\title{
An Inventory of Successful and Unsuccessful Strategies of Pre-Final Engineering Students Tackling a Cloze Test
}

\author{
R. Kalpana* \\ Assistant Professor (Sr.Gr), PSG College of Technology, Coimbatore, India; \\ kalpana.ranganathan@gmail.com
}

\begin{abstract}
The present study is aimed at studying the various strategies used by engineering students attempting a cloze test. A narrative text with 100 blanks was administered to a group of 52 prefinal engineering students. The students were given one-hour time to complete the task. Following the test, a group of students was selected at random and interviewed, and all the interviews were recorded. Select strategies used by the high scorers and low scorers have been identified, classified as appropriate and inappropriate and presented.
\end{abstract}

Keywords: Appropriate and Inappropriate Language Learning Strategies, Cloze Test

\section{Introduction}

The cloze test is a test where every nth word (usually fifth or seventh for a customary total of 50 blanks out of a 250350 word text) is deleted from the text. The cloze test has been used for a wide variety of purposes: to measure reading ability, to measure writing ability, to measure general language proficiency, to relate the relative proficiency of bilingual speakers and to study the relative difficulty of different grammatical items in a text. The present study uses the cloze test as test of reading comprehension and analyses the learning strategies used by learners to tackle the same. It also presents a list of appropriate and inappropriate learning strategies used by learners to comprehend a cloze text.

\section{Review of Literature}

The following paragraphs present a condensed review of literature relevant to the present study, namely, cloze test and learning strategies. Learning strategies refer to operations employed by the learner, which help them in acquiring, storing and retrieving information. They can also refer to "...sets of operations, steps, plans, routines used by learners to facilitate obtaining, storage, retrieval and use of information" [1]. Certain alternative terms used in the place of strategies include tactics, cognitive abilities, functional skills, processing strategies, learning skills, consciously employed operations etc. Developments in cognitive psychology influenced the research on language learning strategies which began in 1960's. The prime concern of all these researchers was on "identifying what good language learners report they do to learn a second or foreign language, or, in some cases, are observed during while learning a second or foreign language" [1]. According to Stern following are the five main languagelearning strategies:

- Management and planning strategies.

- Cognitive strategies.

${ }^{*}$ Author for correspondence 
- Communicative-Experiential Strategies.

- Interpersonal Strategies.

- Affective Strategies.

Rebecca Oxford defines learning strategies as "specific actions taken by the learner to make learning easier, faster, more enjoyable, more self-directed, more effective, and more transferable to new situations" [2]. The following are certain broad classifications of strategies as presented by Rebecca Oxford. Certain strategies are directly involved in the process of language learning and they are known as direct strategies. These direct strategies can be divided into three groups: memory strategies, cognitive strategies and compensation strategies. Memory strategies are those strategies that help learners store and retrieve the material whenever needed. E.g.: grouping. Cognitive strategies are those strategies that help learners to understand and produce new language by different means. E.g.: Summarizing, analyzing, reasoning etc. Compensation strategies help learners to use language though certain knowledge gaps exist in the system of learners. E.g.: Guessing intelligently. Studies on learning strategies have always been a fertile area of research yielding interesting, complex and contradictory results every time.

A cloze test, being a mutilated text, poses a considerable level of challenge to learners. This is because restoring the deletions in such a mutilated text involves both lowerorder and higher-order comprehension skills such as grasping the syntax and word meaning and collocations, identifying tone, following anaphoric and anaphoric references, understanding intersentential relationships and so on. Cloze test, by virtue of its robust nature, is also considered to be an integrative measure of second language proficiency. While tackling a cloze test, the reader has to make use of syntactic and semantic knowledge, and this is fundamental to comprehension. A lot of other intellectual processes like imagining, reasoning, reviewing judging, and problem solving are also essential to any reading task are also involved in attacking a cloze test. A cloze passage makes a lot of demands on the test taker and the most important of them are:

1. Engagement, which means being really very attentive and spending sufficient time on the assignment.

2. Risk-taking, which is the ability to guess the meaning of unknown words, the ability to complete reading a text, the Willingness to answer the gaps, even though one may not be sure of their accuracy.

3. Knowledge which is of three types:
a) Procedural
b) Schemata (formal and content) and

c) Linguistic knowledge

d) Cognitive control [3].

All these factors at times work in combination and at times, they intersect as well. The subjects working on a cloze test should not only be able to understand the type of the text, but also make guesses about the grammatical structures and rhetorical conventions. Only this would help them form intersentential connections and produce vocabulary relevant to the topic. In addition to demonstrating a greater control over structures, learners should coordinate between form and meaning. Basically it is procedural knowledge that keeps readers attempting a cloze test. Comprehension takes place when all stored up knowledge and experiences interact.

While processing a new piece of information or while reading a text, all language learners use learning strategies, either consciously or unconsciously. While reading, or trying to comprehending a text, a reader employs many strategies such as reading, re-reading, linking a particular piece of information with something read earlier and so on. At the core of reading comprehension is the struggle and search for meaning. "Comprehension strategies indicate how readers conceive a task, what textual cues they attend to, how they make sense of what they read, and what they do when they do not understand" [4].

Though many studies have been conducted to find out what strategies learners use and to describe them, this study attempts to spot those specific strategies used by learners to tackle a cloze test. They have been classified as "appropriate" and "inappropriate" strategies - the former referring to those strategies which enable the respondents in achieving the task and the latter referring to those which prevented them getting the right answers. Efforts have also been made to compare and analyze the performance of good and poor scorers and a few interesting findings have been obtained.

The research questions formulated for the study are as follows:

- What are the strategies used by learners to comprehend a cloze test?

- How many strategies thus used enabled the learners to complete the task?

\section{Design of the Experiment}

The objective of the study, as stated earlier, is to identify the strategies used by learners and this has been done by presenting a retrospective account of the reading strategies used by a group of 30 prefinal mechanical engineering 
students. These students were administered a cloze test, a narrative piece that contains 100 blanks. (See Appendix A). The exact scoring method was used for scoring purposes and the raw scores obtained are presented in Table 1. " $R$ " refers to the respondents and the adjacent rows indicate the raw scores obtained by students.

As it can be observed from the table, there are certain tied ranks. With the middle score being 50, 7 respondents who had obtained scores below the midpoint and another 7 respondents who obtained scores above the midpoint were interviewed to find out what strategies were used by these respondents to attempt the task. The interviews did not follow any rigid structure, though the questions were focused to fathom the strategies used by the learners. The investigator uses the "qualitative" or "descriptive" method of analyzing the protocols (the transcribed reports on reading strategies that the subjects produced). Qualitative approaches to research include "ethnography", "nonparticipant observation", "participant observation", "holistic ethnography" and so on and the descriptive method was chosen to allow researchers to make deeper analyses.

\section{Analysis and Discussion}

The following section is an analysis of a few protocols (presented in italics) from the interviews had with the students. The strategies used by the students (both appropriate and inappropriate) are mentioned below as part of the analysis. The researcher made no effort to correct the grammar of the students and the protocols are presented as they were heard and transcribed. For want of space, all the protocols

\begin{tabular}{llll}
\multicolumn{2}{l}{ Table 1. } & & \\
\hline R1 & 80 & R16 & 25 \\
R2 & 75 & R17 & 82 \\
R3 & 16 & R18 & 76 \\
R4 & 23 & R19 & 64 \\
R5 & 34 & R20 & 65 \\
R6 & 43 & R21 & 66 \\
R7 & 52 & R22 & 56 \\
R8 & 34 & R23 & 70 \\
R9 & 62 & R24 & 33 \\
R10 & 11 & R25 & 72 \\
R11 & 54 & R26 & 17 \\
R12 & 73 & R27 & 22 \\
R13 & 12 & R28 & 81 \\
R14 & 62 & R29 & 71 \\
R15 & 62 & R30 & 54 \\
\hline
\end{tabular}

which helped the researcher identify the strategies have not been presented. However, they are listed in Table 2.

S: I have not read such a big passage in one go...that was too much infact... : I Even before I could move from one word to the next, I was afraid, time will be over ..I lost interest reading the last para due to short time

This respondent, as revealed by him, reads very slowly with one eye on the clock. Also, he seems to read and understand each and every word, which is an inappropriate strategy as far as task achievement is concerned.

Though the cloze test demands paying attention to details, it is important that the reader reads in sense groups and understand the overall meaning of the passage and guards himself against getting distracted by particulars. This respondent has obviously not tried to understand the gist of the passage. He did not complete reading the text, which is an inappropriate strategy.

"I like the passage only some what...the title and some words were not very calling...inviting.... I couldn't understand most".

The student respondent does not like the passage because he has not understood it. In such a situation, it is very natural for him to have stopped reading. Thus the student respondent uses the inappropriate strategy of allowing one's feelings to control one's willingness to continue reading.

R: Some answers for the beginning could be written after reading the whole passage. The more you read, the easier it is to understand. Many blanks I filled only after reading seven or eight times.

This student respondent reads and rereads the passage, and this has helped him in filling the blanks. Thus, this appears to be an appropriate reading strategy used by the student to fill in certain blanks.

\section{O: Why did you choose "long" for blank 2?}

\section{R: Just guessed it}

Making uneducated, baseless guesses is an inappropriate strategy. This student respondent probably has not been trained to stimulate her guessing through ample preview questions. She has failed to interrupt her guess and check for its base there, which has made her zero in on the wrong answer.

S: I got "camp" finally only after reading the whole passage. I read the whole thing again for confirming the answer.

Confirming an answer after reading the whole passage is an appropriate strategy used here.

S: Because I was confident "that" was the right answer. No. "That" was right. 
The student respondent is able to recall the correct grammatical structures. Thus, her grammatical knowledge is sufficiently good, though this failed to help her in the later part of the passage where she comes across more complex structures.

\section{O: Why use "boarding" a truck?}

S: Because usually it is boarding a train or bus.

Again, the student respondent arrives at this choice by false analogy i.e. if one can board a bus then one can board a truck too. Thus, he is not paying enough attention to the surrounding context, due to which the fact no truck is going to wait for the refugees to board eludes his understanding.

O: Why did you write "inspector" for blank 21?

S: Judy examined children. So wrote "inspector".

Once again, the student respondent appears to have arrived at this choice by false analogy. She might have been prompted to arrive at this choice because of her experience with school inspectors. These inspectors examine students' books and notebooks when they are on inspection.

\section{O: How did you get "Sudanese"?}

\section{$S:$ It is mentioned earlier.}

Making use of clues and reference words preceding and following the blank is an appropriate strategy used here.

$O$ : You have given the correct construction "put a high priority on". Did you already know the structure?

S: Yes. I knew for sure that the preposition "on" was correct,

Ability to recall and use grammatical structures is an appropriate strategy used by this respondent

S: No. Sanitary facilities are commonly used.

The student respondent is aware of the typical collocations in these word contexts.

O: What were the words you thought would be correct for blank 90?

S: First thought of "black", then "straight". Since she is pouring into a measuring jug thought one "litre" would be right.

Apparently, here the student respondent seems to have done a lot of correct reasoning - making inappropriate guesses in the beginning, but has ended up with a correct guess and reasoning it out-an instance of appropriate strategy.

O: How did you get it as one litre?

S: She was measuring one packet correctly and this has to be mixed with a particular amount of water.

Again this student respondent reasons out correctly, taking into account the contextual clues-one packet and so she is able to reason it out as one litre, which is an appropriate strategy.

O: How did you write "give"? Tell me what are the other words you thought of? How did you arrive at this choice?

S: No I did not think of any other word. "Give" is more general. So I used it. "Feed" did not strike me at all.

Inability to identify the key word is an inappropriate strategy employed here by this student.

S: Yes I liked it. It is something about adventure. How health workers save the civilians. Different from the usual.

This shows that the student respondent is emotionally responding to the text (points the difference in theme). This also indicates that the student respondent is not exposed much to reading this kind of material.

O: How many times did you read it?

S: Only once.

This student respondent has read the passage only once, which is certainly not enough to understand the passage. A passage needs to be read many times at different levels (literal and inferential) for understanding.

$O:$ Is the sentence grammatically ok? "The camp routine was varied"?

S: It is wrong. I did not try to connect it with the following lines.

The student respondent fails to look for grammatical correctness because he had perhaps missed the next word or ignored it because he was not paying enough attention. Hence, the tense is wrongly used. This is an inappropriate strategy.

O: Put a high priority "for". Why use "for"?

S: I thought of "in" but finally used "for".

The student respondent is unable to recall the correct grammatical structure, thus displaying a lack of insufficient linguistic knowledge.

\section{S: I thought of "tank" and "canal" and got confused.}

Again, the student respondent has failed to link what he reads with the content schemata. The student respondent must have stopped to review the material and link it with the content schemata. This is an inappropriate strategy.

S: With blanks 55 and 56, I was not sure of what to use. I did not want to leave anything blank. I just filled in something and did it.... finish it...

This respondent has used wrong words because he does not want to leave anything blank i.e., the student respondent fills in the blank for the sake of filling it - an inappropriate strategy. 
O: Why did you write "weeks"?

S: I thought it was apt. They are caught in the midst of the desert. So, it might take them weeks to reach.

This respondent does not make use of extra-textual cues. He is not able to reason out that it is not possible for one to travel for weeks together in the desert.

O: Why did you choose "that" instead of "which"?

S: I did not think that "that" and "which" make much difference in this context.

The student respondent is unable to recall the grammatical item because at this level, she must have been exposed to the difference between the relative pronouns "that" and "which" - that is, the first one is used with living beings and the second with non-living things.

O: How did you get "Sudanese"?

$S:$ It is mentioned earlier.

Making use of clues and reference words preceding and following the blank is an appropriate strategy used by this respondent.

S: Thought of words like "got", "took". Felt it was not apt. So, I finally used "board".

In this case, the student respondent applies the inappropriate strategy of not filling in words by deducing from the surrounding context.

O: Why did you use "obvious" in blank 62?

S: Used "obvious" in the sense of clear.

Inability to identify the key word "evident" is a problem with vocabulary.

O: Why did you choose yellow "water"?

$S:$ I thought of medicine, where all salts are dissolved in water.

Table 2. Appropriate and Inappropriate Strategies

\begin{tabular}{|c|c|}
\hline Appropriate strategies & Inappropriate strategies \\
\hline $\begin{array}{l}\text { 1. Making use of real world schemata and clues and reference } \\
\text { words preceding and following the blank - an awareness of } \\
\text { anaphoric and cataphoric references. } \\
\text { 2. Filling the easier blanks first i.e. focus on task completion. } \\
\text { 3. Reading and rereading the passage many times for } \\
\text { understanding. } \\
\text { 4. Reading the second half of the passage to fill in the blanks } \\
\text { in the first half - making use of intersentential cues and } \\
\text { discoursal conventions. } \\
\text { 5. Ability to remember references occurring earlier in the } \\
\text { text. } \\
\text { 6. Confirming an answer after reading the whole passage. } \\
\text { 7. Making educated guesses. } \\
\text { 8. Reasoning out the correct answer (which would serve } \\
\text { as a compensation strategy when the required linguistic } \\
\text { knowledge is not present). } \\
\text { 9. Responding to the text emotionally. }\end{array}$ & $\begin{array}{l}\text { 1. Employing false analogy (which might not work } \\
\text { with context-specific texts). } \\
\text { 2. Making uneducated, baseless guesses, overlooking } \\
\text { linguistic and contextual cues. } \\
\text { 3. Failing to tap the content schemata. } \\
\text { 4. Ignoring the extra-textual and linguistic clues. } \\
\text { 5. Skipping a few lines and sentences while reading. } \\
\text { 6. Using wrong words in the blanks just for the sake } \\
\text { of filling them up. } \\
\text { 7. Ignoring the purpose of reading - Reading without } \\
\text { giving in-depth attention. } \\
\text { 8. Responding to the text emotionally. } \\
\text { 9. Failing to pay selective attention. }\end{array}$ \\
\hline
\end{tabular}

Here the student respondent is not reading paying selective attention, which is an inappropriate strategy.

O: Why did you use "the" in blank (9)?

S: Couldn't find any other suitable word.

Many blanks are wrongly filled because she is not able to find suitable words.

O: How did you choose "entirely"?

S: It suited the sentence. Looked only for that sentence to be grammatically correct did not look for meaning.

O: How did you find the passage to be?

$S$ : When read for the first time, could not form any clear idea. Some places very difficult to find the right word.

The only appropriate strategy used by this student respondent is making use of clues and references preceding and following the blank. This student appears to have a lot of problems with grammar and also with vocabulary.

The table in the next page (Table 2.) presents a few strategies used by students as presented in the analysis. This table includes both the appropriate and the inappropriate strategies. Interestingly, certain strategies such as emotionally responding to the text and recalling real -world schemata occurs in both the categories and offers sufficient thought on the impact of "affect" on the task achievement.

\section{Conclusion}

In summary, the analyses presented in the previous section offers a peep into the workings of various strategies by learners. However, the study does have certain limitations. 
Firstly, the list of strategies reported here are by no means exhaustive. They are just a sample of whatever the researcher could decipher from the interviews she had with the respondents. Yet another limitation of the study is that mentalistic study of strategies is always a fuzzy area and it should also be remembered that many of these students must have used a lot of "hidden" or unconscious strategies. Most respondents found the task to be difficult and challenging one and it naturally follows that they must have used a lot of strategies. However, only those that were consciously revealed have been mentioned. The other unconscious or "hidden" strategies are very difficult for an observer to discover and analyse. This is the problem faced by any researcher trying to analyse strategies. Generally, observation scales miss the mentalistic strategies. Choosing correct observation scales and forming one's own observation form by making a list of important strategies, which one thinks are important and used often, can yield more information. Thinkaloud interviews, semi-structured interviews, self report surveys, diaries and journals and selected combinations of the above-mentioned tools could provide deeper insights into the strategies deployed. Also, the sample is not representative of the entire population of engineering students attempting a cloze test. Lastly, the use of the term "appropriate" and "inappropriate" does echo a certain degree of subjectivity and judgment. However, the researcher has used the term for want of suitable technical equivalents in literature.

\section{Works Cited}

1. Wenden A., Rubin J., Learner strategies in language learning, New Jersey: Prentice Hall, 1987.

2. Oxford L.R., Language Strategies: What Every Teacher Should Know, New York: Newbury House Publishers, 1990.

3. Vann R.J., Abraham R.G., "Strategies of unsuccessful Language Learners”, TESOL Quarterly, 24(2):177-180, 1990.

4. Block E., "The Comprehension Strategies of Second Language Readers”, TESOL Quarterly, vol. 20(3), pp. 463-494, 1986

\section{Other References}

1. Van Parrernen C.F., Van Parrernen M.C.S., "Contextual Guessing: A Trainable Reader Strategy”, System, vol. 9(3), pp. 235-241, 1981.

2. Smith F., Understanding Reading: A Psycholinguistic Analysis of Reading and Learning to Read, 5th ed, New Jersey: Erlbaum Associates, 1994.

\section{Appendix A}

Name of the Test-Taker :

Batch

Date

Start Time

Finish Time

\section{Instructions}

Read the entire passage carefully before filling in the blanks. If you are not sure of a word, do not be afraid to guess the word. When you guess check whether the work fits the overall sense of the passage as well as the sentence in which it occurs. There are 100 blanks in this passage. Each blank has to be filled in with one word. You have about $100 \mathrm{~min}$ utes for filling up the blanks. Use a ball pen or ink pen to write the answers. Do not use a pencil. Write legibly.

Judy examined Amaresh and shook her head in wonder and frustration. It had taken Amaresh (1) ___ her three children (2) to make the terrifying (3) from their Ethiopian village (4)

Tigre to the refugee (5) in the desert across (6) Sudanese border. They had (7)

the whole way, hiding (8) the day and traveling (9) at night to avoid (10) bombs and bullets of (11) Ethiopian military planes. They (12) their journey with only (13) small amount of food, (14) had been used up (15) before they reached the (16) border.

The trucks they (17) at the border took (18) directly to the refugee (19) camp's volunteer health (21) as one of (20) __ camps vol vamined many such (22) as they arrived weak (23) starvation and fatigue. She (24) Amaresh's two youngest children (25) combat their severe special feeding program (26) malnutrition (27) then showed the family (28) new home - a single 10 (29) by 10 foot tent (30) already housed 15 of (31) relatives.

The camp routine (32) varied. Each day Amaresh (33) to collect her family's (34) of raw wheat, beans cooking oil. She mixed (36) together and boiled them (37) the blackish green water drawn (38) ___ the camp's water tanks. (39) recently water had been (40) directly from a nearby (41) canal, and diarrhea had rampant in the camp. (43) 
many of the refugees (44) this water were so (45) by malnutrition that even (46)

simple diarrhea was life threatening - (47) the children and (48) elderly. The health workers (49) put a high priority (50) construction of the water (51)___ which allowed the canal (52) to be chlorinated and (53) settle before being used in (54) camp.

The water was (55) the only source (56) of for the health workers. (57) camp had no sanitary (58) All of the camp's 12,000 (59) used the open field (60) to the camp, when (64) fierce sandstorm destroyed most (65) the tents and the (66) water system. The only (67) water was that pumped (68) from the canal, without (69)

chlorination or settling. By (70) next morning, with the (71) rising to 110 degrees $\mathrm{F}$, everyone (72) the camp was suffering (73) terrible diarrhea. Judy was (74) surprised when Amaresh came (75) her that evening carrying (76) youngest son. He was (77) pale and unresponsive and (78) obviously suffering from severe (79) from his diarrhea. Since (80) were no antibiotics available, (81) gave Amaresh a yellow (82) jug and several small (83) packets containing oral dehydration (84) (ORS). Judy instructed Amaresh to (85) the white powder from (86) packet into the jug (87) then to fill the (88) with chlorinated water up (89) ___ a clearly marked one (90)___ line. She was to (91) as much of this (92) to her children as (93) could drink. Judy predicted (94) the children would be (95) playing with their friends (96) only a few days. (97) that (98) oral rehydration salts (99) distributed have become almost miracle treatment for refugees (100) from dehydration caused by diarrhea. It was remarkable that in the six weeks Judy had worked at the camp not one child had died from dehydration with diarrhea!

Thank you for Attempting this Test!

Table 3. Key (score using the exact word method: will not discriminate between any two almost equally language proficient candidates)

\begin{tabular}{|c|c|c|c|c|}
\hline 1. and & 21. workers & 41. irrigation & 61. danger & 81. she \\
\hline 2. days & 22. families & 42. been & 62. obvious & 82. plastic \\
\hline 3. journey & 23. with & 43. unfortunately & 63. arrival & 83. sealed \\
\hline 4. in & 24. assigned & 44. drinking & 64. a & 84. salts \\
\hline 5. camp & 25. to & 45. weakened & 65. of & 85. pour \\
\hline 6. the & 26. to & 46. such & 66. camp's & 86. a \\
\hline 7. walked & 27. and & 47. especially & 67. available & 87. and \\
\hline 8. during & 28. their foot & 48. the & 68. directly & 88. jug \\
\hline 9. only & 29. that & 49. had & 69. any & 89. to \\
\hline 10. the & 30. their & 50. on & 70. the & 90. litre/liter \\
\hline 11. the & 31. never & 51. tanks & 71. temperature & 91. feed \\
\hline 12. started & 32. went & 52. water & 72. in & 92. drink \\
\hline 13. a & 33. allotment & 53. to & 73. from & 93. they \\
\hline 14. which & 34. allotment & 54. the & 74. not & 94. that \\
\hline 15. long & 35. and & 55. not & 75. to & 95. out \\
\hline 16. sudanese & 36. these & 56. worry & 76. her & 96. in \\
\hline 17. met & 37. in & 57. the & 77. very & 97. the \\
\hline 18. them & 38. from & 58. facilities & 78. was & 98. Judy \\
\hline 19. camp & 39. until & 59. refugees & 79. dehydration & 99. A \\
\hline 20. the & 40. taken & 60. next & 80. there & 100. suffering \\
\hline
\end{tabular}

